\title{
Equivalence between intersection cuts and the corner polyhedron
}

\author{
Michele Conforti \\ Università di Padova, conforti@math.unipd.it \\ Gérard Cornuéjols * \\ Carnegie Mellon University, gc0v@andrew.cmu.edu \\ Giacomo Zambelli \\ Università di Padova, giacomo@math.unipd.it
}

November 2009, revised January 2010

\begin{abstract}
Intersection cuts were introduced by Balas and the corner polyhedron by Gomory. Balas showed that intersection cuts are valid for the corner polyhedron. In this paper we show that, conversely, every nontrivial facet-defining inequality for the corner polyhedron is an intersection cut.
\end{abstract}

Keywords: integer programming, cutting plane, corner polyhedron, intersection cut

We consider a mixed integer linear set

$$
\begin{array}{ll}
A x=b & \\
x_{j} \in \mathbb{Z} & \text { for } j=1, \ldots, p \\
x_{j} \geq 0 & \text { for } j=1, \ldots, n
\end{array}
$$

where $p \leq n, A$ is a matrix in $\mathbb{Q}^{m \times n}$, and $b$ is a column vector in $\mathbb{Q}^{m}$. We assume that $A$ has full row rank $m$. Given a feasible basis $B$, let $N=\{1, \ldots, n\} \backslash B$ index the nonbasic variables. We rewrite the system $A x=b$ as

$$
x_{i}=\bar{b}_{i}-\sum_{j \in N} \bar{a}_{i j} x_{j} \quad \text { for } i \in B
$$

where $\bar{b}_{i} \geq 0, i \in B$.

The corner polyhedron introduced by Gomory [8] is obtained from (1) by dropping the nonnegativity restriction on all the basic variables $x_{i}, i \in B$, in (2). Note that in this

\footnotetext{
${ }^{*}$ Corresponding author: Tepper School of Business, Carnegie Mellon University, 5000 Forbes Avenue, Pittsburgh, PA 15213.
} 
relaxation we can drop the constraints $x_{i}=\bar{b}_{i}-\sum_{j \in N} \bar{a}_{i j} x_{j}$ for all $i \in B \cap\{p+1, \ldots, n\}$ since these variables $x_{i}$ are continuous and only appear in one equation and no other constraint. Therefore from now on we assume that all basic variables in (2) are integer variables, i.e. $B \subseteq\{1, \ldots, p\}$.

Therefore the relaxation of (1) introduced by Gomory is the mixed integer set

$$
\begin{array}{ll}
x_{i}=\bar{b}_{i}-\sum_{j \in N} \bar{a}_{i j} x_{j} & \text { for } i \in B \\
x_{i} \in \mathbb{Z} & \text { for } i=1, \ldots, p \\
x_{j} \geq 0 & \text { for } j \in N .
\end{array}
$$

The convex hull of the feasible solutions to (3) is called the corner polyhedron relative to the basis $B$ and it is denoted by $\operatorname{corner}(B)$.

Let $P(B)$ be the linear relaxation of $(3) . P(B)$ is an $|N|$-dimensional polyhedron with a unique vertex, namely $\bar{x}_{i}=\bar{b}_{i}$, for $i \in B, \bar{x}_{j}=0$, for $j \in N$, and with $|N|$ extreme rays, $\bar{r}^{j}$ for $j \in N$, defined by

$$
\bar{r}_{h}^{j}= \begin{cases}-\bar{a}_{h j} & \text { if } h \in B, \\ 1 & \text { if } j=h, \\ 0 & \text { if } h \in N \backslash\{j\} .\end{cases}
$$

The corner polyhedron has been investigated over the last few decades. It is well known, and easy to prove, that corner $(B)$ is nonempty if and only if there exists a point in $\mathbb{Z}^{p} \times \mathbb{R}^{n-p}$ satisfying $x_{i}=\bar{b}_{i}-\sum_{j \in N} \bar{a}_{i j} x_{j}$ for all $i \in B$. Furthermore, if $\operatorname{corner}(B)$ is nonempty, then it is $|N|$-dimensional, and its extreme rays are $\bar{r}^{j}$ for $j \in N$. We will assume throughout this note that $\operatorname{corner}(B)$ is nonempty.

Clearly, $P(B)$ coincides with $\operatorname{corner}(B)$ when $\bar{x}_{i} \in \mathbb{Z}$ for $i=1, \ldots, p$. If this is not the case, $\bar{x}$ does not belong to corner $(B)$ and we address the problem of finding valid inequalities for the set (1) that are violated by the point $\bar{x}$. Balas [2] proposed the following construction to generate valid inequalities for the corner polyhedron that cut off the basic solution $\bar{x}$.

Consider a closed convex set $C \subseteq \mathbb{R}^{n}$ such that the interior of $C$ contains the point $\bar{x}$. Assume that the interior of $C$ contains no point in $\mathbb{Z}^{p} \times \mathbb{R}^{n-p}$. For $j \in N$, define

$$
\alpha_{j}=\max \left\{\alpha \geq 0: \bar{x}+\alpha \bar{r}^{j} \in C\right\} .
$$

Since $\bar{x}$ is in the interior of $C, \alpha_{j}>0$. When $r_{j}$ belongs to the recession cone of $C$, we have $\alpha_{j}=+\infty$. Define $\frac{1}{+\infty}=0$. The inequality

$$
\sum_{j \in N} \frac{x_{j}}{\alpha_{j}} \geq 1
$$

is the intersection cut defined by $C$.

Balas [2] showed that intersection cuts are valid for corner $(B)$. The following theorem provides a converse statement, namely that corner $(B)$ is defined by the intersection cuts.

A valid inequality for corner $(B)$ is trivial if it is implied by the nonnegativity constraints $x_{j} \geq 0, j \in N$. Every nontrivial valid inequality for corner $(B)$ can be expressed in terms of the nonbasic variables only, and can be written in the form $\sum_{j \in N} \gamma_{j} x_{j} \geq 1$, where $\gamma_{j} \geq 0$ for all $j \in N$. We say that such an inequality is minimal if there is no other valid inequality 
$\sum_{j \in N} \gamma_{j}^{\prime} x_{j} \geq 1$ for corner $(B)$ such that $\gamma_{j}^{\prime} \leq \gamma_{j}$ for all $j \in N$, and the inequality is strict for at least one index $j \in N$. We say that it is rational if $\gamma_{j} \in \mathbb{Q}$ for all $j \in N$. Since $A$ and $b$ are rational, every nontrivial facet-defining inequality for $\operatorname{corner}(B)$ is rational and minimal.

Theorem 1. If corner $(B)$ is nonempty, every nontrivial rational minimal valid inequality for $\operatorname{corner}(B)$ is an intersection cut.

Proof. Assume that corner $(B)$ is nonempty, and let $\sum_{j \in N} \gamma_{j} x_{j} \geq 1$ be a rational minimal valid inequality for corner $(B)$. Consider the simplex

$$
S=\left\{x \in \mathbb{R}^{n} \mid \sum_{j \in N} \gamma_{j} x_{j} \leq 1, x_{j} \geq 0 \text { for } j \in N, x_{i}=\bar{b}_{i}-\sum_{j \in N} \bar{a}_{i j} x_{j} \text { for } i \in B\right\} .
$$

We will enlarge $S$ into a convex set $C$ containing $\bar{x}$ in its interior but no point of $\mathbb{Z}^{p} \times \mathbb{R}^{n-p}$, and observe that $\sum_{j \in N} \gamma_{j} x_{j} \leq 1$ is the intersection cut defined by $C$. This is straightforward in the pure integer case, since we only need to relax the inequalities $x_{j} \geq 0, j \in N$. The crux of the proof is how to do this in the mixed integer case. We first project $S$ onto the space of integer variables (note that any polytope may arise as the projection of a simplex), then we enlarge it to a set $K$ and construct a cylinder over $K$.

(1) No face $F$ of $S$ containing $\bar{x}$ has a point of $\mathbb{Z}^{p} \times \mathbb{R}^{n-p}$ in its relative interior (including the improper face $F=S$ ).

Indeed, let $\tilde{x}$ be a point of $S$ in $\mathbb{Z}^{p} \times \mathbb{R}^{n-p}$. Since $S \subseteq P(B), \tilde{x}$ belongs to corner $(B)$ and since $\sum_{j \in N} \gamma_{j} x_{j} \geq 1$ is a valid inequality for corner $(B)$, then $\sum_{j \in N} \gamma_{j} \tilde{x}_{j}=1$.

Let $F$ be a face of $S$ containing $\bar{x}$, and suppose that $\tilde{x}$ is in the relative interior of $F$. Then there exists a scalar $\lambda>1$ such that $z=\bar{x}+\lambda(\tilde{x}-\bar{x})$ is in $F$. Since $\bar{x}_{j}=0, j \in N$ and $\sum_{j \in N} \gamma_{j} \tilde{x}_{j}=1$, then $\sum_{j \in N} \gamma_{j} z_{j}>1$. This contradicts the fact that $z \in S$ and (1) is proven.

Let $\tilde{S}=S+L$ where $L=\{0\}^{p} \times \mathbb{R}^{n-p}$. Since $S$ is a rational polyhedron and the lineality space of $\tilde{S}$ contains $L$, the polyhedron $\tilde{S}$ can be expressed as $\tilde{S}=\left\{x \in \mathbb{R}^{n} \mid \sum_{j=1}^{p} c_{j}^{i} x_{j} \leq\right.$ $\left.d_{i}, i=1, \ldots, t\right\}$ for some $c^{1}, \ldots, c^{t} \in \mathbb{Z}^{p}$ and $d_{1}, \ldots, d_{t} \in \mathbb{Z}$. (Indeed $\left\{x \in \mathbb{R}^{p} \mid \sum_{j=1}^{p} c_{j}^{i} x_{j} \leq\right.$ $\left.d_{i}, i=1, \ldots, t\right\}$ is the projection of $S$ onto $\mathbb{R}^{p}$.)

(2) No face of $\tilde{S}$ containing $\bar{x}$ has a point of $\mathbb{Z}^{p} \times \mathbb{R}^{n-p}$ in its relative interior.

Let $\tilde{F}$ be a face of $\tilde{S}$ and let $F=S \cap \tilde{F}$. Then $F$ is a face of $S$ and $\tilde{F}=F+L$. Therefore, since $L$ is in the lineality space of $\tilde{F}$, we have $\operatorname{relint}(\tilde{F})=\operatorname{relint}(F)+L$, where $\operatorname{relint}(\cdot)$ denotes the relative interior of a set. Assume $\tilde{F}$ contains $\bar{x}$. Since $\bar{x}$ belongs to $S$ and $F=S \cap \tilde{F}$, we have $\bar{x} \in F$. Assume $\operatorname{relint}(\tilde{F})$ contains $\tilde{x}$ in $\mathbb{Z}^{p} \times \mathbb{R}^{n-p}$. Then $\tilde{x}+L$ is contained in $\operatorname{relint}(\tilde{F}) \cap\left(\mathbb{Z}^{p} \times \mathbb{R}^{n-p}\right)$. Since $\operatorname{relint}(\tilde{F})=\operatorname{relint}(F)+L$, we have $\tilde{x}+L$ contains a point in $\operatorname{relint}(F) \cap\left(\mathbb{Z}^{p} \times \mathbb{R}^{n-p}\right)$, a contradiction to (1). This proves $(2)$.

(3) There exists a convex set $K \subset \mathbb{R}^{p}$ with no point of $\mathbb{Z}^{p}$ in its interior such that the set $C:=K \times \mathbb{R}^{n-p}$ contains $\bar{x}$ in its interior and $\tilde{S} \subseteq C$.

Assume, without loss of generality, that $\bar{x}$ satisfies at equality the first $h$ constraints defining $\tilde{S}$ (possibly $h=0$ ), and none of the other constraints. That is

$$
\begin{aligned}
& \sum_{j=1}^{p} c_{j}^{i} \bar{x}_{j}=d_{i} \quad i=1, \ldots, h \\
& \sum_{j=1}^{p} c_{j}^{i} \bar{x}_{j}<d_{i} \quad i=h+1, \ldots, t
\end{aligned}
$$


Define $d_{i}^{\prime}=d_{i}+1$ for $i=1, \ldots, h$ and $d_{i}^{\prime}=d_{i}$ for $i=h+1, \ldots, t$, and let $K=\{x \in$ $\left.\mathbb{R}^{p} \mid \sum_{j=1}^{p} c_{j}^{i} x_{j} \leq d_{i}^{\prime}, \ldots, i=1, \ldots, t\right\}$ and $C=K \times \mathbb{R}^{n-p}$. Note that $C=\left\{x \in \mathbb{R}^{n} \mid \sum_{j=1}^{p} c_{j}^{i} x_{j} \leq\right.$ $\left.d_{i}^{\prime}, \ldots, i=1, \ldots, t\right\}$. By construction, $\bar{x}$ is in the interior of $C$ and $\tilde{S} \subseteq C$.

We only need to show that $K$ contains no point of $\mathbb{Z}^{p}$ in its interior. Suppose not. Then there exists $\tilde{x} \in \mathbb{Z}^{p} \times \mathbb{R}^{n-p}$ such that $\tilde{x}$ is in the interior of $C$. Hence $\sum_{j=1}^{p} c_{j}^{i} \tilde{x}_{j}<d_{i}^{\prime}$ for $i=1, \ldots, t$. By definition of $d^{\prime}$ and since $\tilde{x}_{j} \in \mathbb{Z}$ for $j=1, \ldots, p$, we have $\sum_{j=1}^{p} c_{j}^{i} \tilde{x}_{j} \leq d_{i}$ for $i=1, \ldots, h$, and $\sum_{j=1}^{p} c_{j}^{i} \tilde{x}_{j}<d_{i}$ for $i=h+1, \ldots, t$. Let $J$ be the set of indices $i$ such that $\sum_{j=1}^{p} c_{j}^{i} \tilde{x}_{j}=d_{i}$, and $\tilde{F}=\left\{x \in \tilde{S} \mid \sum_{j=1}^{p} c_{j}^{i} \tilde{x}_{j}=d_{i}, i \in J\right\}$. By construction, $\tilde{F}$ is a face of $\tilde{S}$ containing $\bar{x}$, and $\tilde{x}$ is a point of $\mathbb{Z}^{p} \times \mathbb{R}^{n-p}$ in the relative interior of $\tilde{F}$, a contradiction to (2) and this proves (3).

Let $\sum_{j \in N} \alpha_{j}^{-1} x_{j} \geq 1$ be the intersection cut defined by $C$, where $\alpha_{j}$ is defined by (5) for all $j \in N$. Since $\bar{x}+\gamma_{j}^{-1} \bar{r}^{j} \in S$ and $S \subseteq C$, the definition of $\alpha_{j}$ implies $\gamma_{j}^{-1} \leq \alpha_{j}, j \in N$. By minimality of $\sum_{j \in N} \gamma_{j} x_{j} \geq 1$, we have $\gamma_{j} \leq \alpha_{j}^{-1}$, thus $\gamma_{j}=\alpha_{j}$ for all $j \in N$.

We conclude this note with two consequences. The convex sets needed to generate minimal intersection cuts are of the form $K \times \mathbb{R}^{n-p}$, where $K$ is a maximal lattice-free convex set in $\mathbb{R}^{p}$ (lattice-free means that $K$ does not contain any point of $\mathbb{Z}^{p}$ in its interior). A theorem of Lovász [10] states that maximal lattice-free convex sets are polyhedra.

An attractive feature of minimal intersection cuts is that they are given by "formulas" depending only on the number $p$ of integer variables and the vector $\bar{b} \in \mathbb{R}^{|B|}$. Indeed, let $\tilde{x} \in \mathbb{R}^{p}$ be defined by $\tilde{x}_{i}=\bar{x}_{i}, i=1, \ldots, p$. Given a lattice-free convex set $K \subset \mathbb{R}^{p}$ containing $\tilde{x}$ in its interior, let $\gamma_{K}: \mathbb{R}^{p} \rightarrow \mathbb{R}$ be the gauge of $K-\tilde{x}$, which is defined by

$$
\gamma_{K}(r)=\inf \left\{t \mid \tilde{x}+t^{-1} r \in K\right\}, \quad r \in \mathbb{R}^{p} .
$$

Then the intersection cut defined by $K \times \mathbb{R}^{n-p}$ is $\sum_{j \in N} \gamma_{K}\left(\tilde{r}^{j}\right) s_{j} \geq 1$, where $\tilde{r}^{j} \in \mathbb{R}^{p}$ is defined by $\tilde{r}_{i}^{j}=\bar{r}_{i}^{j}, i=1, \ldots, p$. Note that the above function $\gamma_{K}$ gives a valid inequality for any choice of the coefficients $\bar{a}_{i j}$ and for any number of continuous variables.

A function $\psi: \mathbb{R}^{p} \rightarrow \mathbb{R}$ is a valid function if $\sum_{j \in N} \psi\left(\tilde{r}^{j}\right) x_{j} \geq 1$ is valid for $\operatorname{corner}(B)$ for any choice of the $\bar{a}_{i j}$ s and for any number of continuous variables. Our theorem, together with a result of Borozan and Cornuéjols [5], implies that all valid functions are dominated by functions of the form $\gamma_{K}$, for some lattice-free convex set $K$, and furthermore every inequality of corner $(B)$ is generated by some valid function. This proves the following.

Corollary 2. Every minimal valid function is continuous and piecewise linear.

This is related to a conjecture of Gomory and Johnson [9], stating that all valid functions for the infinite group problem are piecewise linear. This conjecture was disproved in [4], although the above corollary shows that the statement holds when the number of integer variables is fixed.

A nontrivial result in integer programming is that, for a mixed integer linear set (1) with rational data, the split closure is a polyhedron (Cook, Kannan and Schrijver [6]; alternate proofs are presented in [1], [7] and [11]). The split closure is the set obtained from all intersection cuts generated from all bases (feasible and infeasible) and all split disjunctions [1]. 
More generally, define the intersection closure of a mixed integer linear set as the set obtained from all intersection cuts generated from all bases (feasible and infeasible) and all lattice-free convex sets. Theorem 1 implies that, for a mixed integer linear set with rational data, the intersection closure is the intersection of a finite number of corner polyhedra. Therefore the intersection closure is a polyhedron.

Corollary 3. The intersection closure of a rational mixed integer linear set is a polyhedron.

Acknowledgements: We thank the associate editor for his excellent suggestions. Michele Conforti and Giacomo Zambelli's research was supported by the Progetto di Eccellenza 20082009 of the Fondazione Cassa di Risparmio di Padova e Rovigo. Gérard Cornuéjols' research was supported by NSF grant CMMI0653419, ONR grant N00014-09-1-0133 and ANR grant ANR06-BLAN-0375.

\section{References}

[1] K. Andersen, G. Cornuéjols, Y. Li, Split Closure and Intersection Cuts, Mathematical Programming A 102 (2005) 457-493.

[2] E. Balas, Intersection Cuts - A New Type of Cutting Planes for Integer Programming, Operations Research 19 (1971) 19-39.

[3] E. Balas, Disjunctive Programming: Properties of the Convex Hull of Feasible Points, GSIA Management Science Research Report MSRR 348, Carnegie Mellon University (1974), published as invited paper in Discrete Applied Mathematics 89 (1998) 1-44.

[4] A. Basu, M. Conforti, G. Cornuéjols, G. Zambelli, A Counterexample to a Conjecture of Gomory and Johnson, manuscript (2008).

[5] V. Borozan, G. Cornuéjols, Minimal Valid Inequalities for Integer Constraints, Mathematics of Operations Research 34 (2009) 538-546.

[6] W.J. Cook, R. Kannan, A. Schrijver, Chvátal Closures for Mixed Integer Programming Problems, Mathematical Programming A 47 (1990) 155-174.

[7] S. Dash, O. Günlük, A. Lodi, MIR Closures of Polyhedral Sets, Mathematical Programming A 121 (2010) 33-60.

[8] R.G. Gomory, Some Polyhedra Related to Combinatorial Problems, Linear Algebra and Applications 2 (1969) 451-558.

[9] Gomory, R. E., Johnson, E. L., T-space and Cutting Planes, Mathematical Programming, Series B 96 (2003) 341-375.

[10] L. Lovász, Geometry of Numbers and Integer Programming, Mathematical Programming: Recent Developements and Applications, M. Iri and K. Tanabe eds., Kluwer (1989) 177-210. 
[11] J.P. Vielma, A Constructive Characterization of the Split Closure of a Mixed Integer Linear Program, Operations Research Letters 35 (2007) 29-35. 